\title{
Reduced polarity and improved dispersion of microfibrillated cellulose in poly(lactic-acid) provided by residual lignin and hemicellulose
}

\author{
A. Winter ${ }^{1}$, L. Andorfer ${ }^{1}$, S. Herzele ${ }^{2}$, T. Zimmermann ${ }^{3}$, B. Saake ${ }^{4}$, M. Edler ${ }^{5}$, T. Griesser ${ }^{5}$, J. Konnerth ${ }^{1}$, and \\ W. Gindl-Altmutter ${ }^{1, \star}$ \\ ${ }^{1}$ Department of Materials Science and Process Engineering, BOKU-University of Natural Resources and Life Science, Vienna, Austria \\ ${ }^{2}$ Kompetenzzentrum Holz GmbH, Linz, Austria \\ ${ }^{3}$ Applied Wood Materials Laboratory, Empa, Swiss Federal Laboratories for Materials Science and Technology, Dübendorf, \\ Switzerland \\ ${ }^{4}$ Zentrum Holzwirtschaft, University of Hamburg, Hamburg-Bergedorf, Germany \\ ${ }^{5}$ Department of Polymer Technology, University of Leoben, Leoben, Austria
}

Received: 20 July 2016

Accepted: 22 September 2016

Published online:

29 September 2016

(C) The Author(s) 2016. This article is published with open access at Springerlink.com

\begin{abstract}
The surface chemistry and dispersion in poly(lactic-acid) of microfibrillated wood and microfibrillated lignocellulose prepared from untreated and partially delignified beech were compared with conventional microfibrillated cellulose produced from bleached pulp. High heterogeneity in fibril morphology and bulk chemical composition was observed. Also surface chemistry of the fibrils was highly variable, but not clearly correlated with bulk chemistry. Composite solution-cast films of poly(lacticacid) reinforced with $1 \%$ fibrils were produced by adding fibrils dried from solvent into a polymer solution. Highly variable dispersion of fibrils correlated with varying mechanical performance was observed. Correlations were obtained between surface chemistry of fibrils as revealed by X-ray photoelectron spectroscopy and adhesion force microscopy on the one hand and the tensile performance of the fibril-reinforced polymer composites on the other hand. Overall, certain variants of fibrillated material with residual lignin and hemicellulose content showed reduced surface polarity, improved dispersion in poly(lactic-acid) and improved reinforcement efficiency compared to conventional MFC produced from bleached pulp.
\end{abstract}

\section{Introduction}

Microfibrillated cellulose (MFC) is one of the most promising raw materials for the design of future biobased alternatives to current fossil-based structural materials. Its discovery has boosted innovation in bio-based fibre-reinforced polymer composites [1-4]. Compared to other cellulosic fibres such as flax, hemp, or sisal, and regenerated cellulose fibres, MFC excels in mechanical performance due to its semi-

Address correspondence to E-mail: wolfgang.gindl@boku.ac.at 
crystalline cellulosic structure and high specific surface area. MFC is easily processed in aqueous media due to the essentially hydrophilic nature of cellulose, which is rich in accessible-OH groups. Even though the cellulose I crystal also disposes of nonpolar moieties [5], processing of MFC in hydrophobic media usually requires chemical surface modification to impart compatibility with organic solvents and hydrophobic polymers [6]. Since chemical surface modification adds significant additional cost to MFC, it is one factor hindering commercialisation of this novel bio-based nano-reinforcement fibre.

In the native wood cell wall cellulose microfibrils serve as reinforcement to a perfectly functional and naturally optimised composite, where they are embedded in a lignin-hemicellulose matrix. It is widely accepted that hemicellulose acts similar to a coupling agent linking hydrophilic cellulose with the mostly hydrophobic aromatic cell-wall polymer lignin [7]. In view of this biogenic model system, the question arises whether the compatibilising function of hemicellulose and the hydrophobic moieties present in lignin can be utilised towards providing surface-chemical compatibility between polar MFC and nonpolar polymers, similar to a coupling agent. The beneficial role of residual hemicellulose in MFC with regard to compatibilisation with nonpolar media has been highlighted under the term microfibrillated holocellulose. It was shown that this material yields nanopaper structures of high quality and optical transparency $[8,9]$. Residual hemicellulose in fibrillated holocellulose enables improved dispersion of MFC in non-polar solvent [10]. Moreover, holocellulose nanocrystals produced by acid hydrolysis are amphiphilic in surface-chemical terms and capable of stabilising oil-in-water emulsions [11].

Similarly to hemicellulose, residual lignin in MFC was proposed to impart advantageous characteristics in terms of improved compatibility [12]. It was observed that lignin-containing MFC (microfibrillated lignocellulose, MFLC) shows higher fineness of fibrils compared to MFC prepared from bleached pulp [13, 14], presumably due to the radical scavenging property of lignin. Also, MFLC exhibited less hydrophilicity compared to MFC, and was well miscible with nonpolar solvents [14, 15]. When compounded with hydrophobic polymeric matrices, MFLC showed better dispersion and improved reinforcement efficiency compared to MFC [14-18].
The results found in literature to date indicate a clear potential for MFLC to provide polymer reinforcement with inherent surface chemical compatibility to certain hydrophobic matrices. Motivated by these apparent advantages of MFLC over MFC, we present a systematic survey of variability in surface chemistry and resulting dispersion in poly(lacticacid) (PLA) comparing fibrillated wood, partially delignified wood, and fully de-lignified MFC. PLA is one of the most promising thermoplastic biopolymers in view of replacing or complementing fossil-based polymers in certain applications [19], and therefore a suitable model polymer in the context of the present study.

\section{Materials and methods}

\section{Preparation of fibrillated material}

Microfibrillated lignocellulose (MFLC): Bark-free beech wood chips were partly delignified in a water/ ethanol (50/50 weight) mixture (ethanol with purity of $96.6 \%$ denatured with $1 \%$ petroleum ether, Brüggemann Alkohol, Heilbronn, Germany) in batches of $6 \mathrm{~kg}$ (dry mass). At a mass ratio of 1:4 (wood/solvent), variants as shown in Table 1 were processed in an autoclave. Sulfuric acid was added to catalyse delignification and enabling pulping at lower temperature, pressure and reaction time compared to the system without catalyst. Based on the previous results a progressive lignin removal from variant MFLC1 to MFLC3 could be achieved by addition of $0,0.25$ and $0.75 \%$ sulfuric acid (95\%, Fischer Scientific, Schwerte, Germany) based on the dry weight of wood [20]. Several studies indicate that sulfuric acid accelerates delignification in an organosolv process [21]. Compared to these variants, treatment of MFLC4 was presumed to very mild due to reduced time spent in the reactor. After the defined duration of treatment, the reactor was cooled to $40{ }^{\circ} \mathrm{C}$ and the material was washed with the same 50/50 water/ethanol mixture used for treatment to remove dissolved substances, in particular lignin. After three additional washing cycles in water the material was processed into fibres in a disc refiner. Excess water was removed by centrifugation and the material was refrigerated at $-20{ }^{\circ} \mathrm{C}$ before further usage. For fibrillation, the material was left to thaw and swell overnight in deionised water at room temperature. 
Table 1 Treatment parameters for partial delignification in water/ethanol

\begin{tabular}{lllll}
\hline Specimen type & Temperature $\left({ }^{\circ} \mathrm{C}\right)$ & Pressure (bar) & $\mathrm{H}_{2} \mathrm{SO}_{4}(\%)$ & Duration (min) \\
\hline MFLC 1 & 170 & 15 & 0 & 90 \\
MFLC 2 & 170 & 15 & 0.25 & 90 \\
MFLC 3 & 170 & 15 & 0.75 & 90 \\
MFLC 4 & 170 & 15 & 0 & 60 \\
\hline
\end{tabular}

Microfibrillated wood (MFW): In parallel to the preparation of MFLC, untreated beech wood chips from the same lot were milled in a rotary mill to a particle size $<1 \mathrm{~mm}$ and also left to swell overnight. Fibrillation was carried out with five passes through a Masuko Supermasscolloider (MKCA6-2J, Masuko Sangyo, Japan) at $1500 \mathrm{rpm}$ and a nominal gap clearance of $-50 \mu \mathrm{m}$. Note that despite setting a negative nominal clearance a gap forms during operation of the grinder because of the hydrodynamic pressure of MFC slurry exiting from the space between the two rotating discs of the grinder.

Microfibrillated cellulose (MFC): Conventional microfibrillated cellulose was obtained from the University of Maine (http://umaine.edu/pdc/pro cess-and-product-development/selected-projects / nanocellulose-facility/) and used as-received. The material is produced without pre-treatment from bleached softwood Kraft pulp using a mechanical refiner.

\section{Characterisation of fibrillated material}

The lignin content of the different variants of fibrillated material was determined gravimetrically from the dry residue after total acid hydrolysis of carbohydrates. The first stage of hydrolysis was performed in $72 \mathrm{w} \%$ concentrated sulfuric acid (72 w\%, Merck, Darmstadt) at $30{ }^{\circ} \mathrm{C}$ for 60 minutes The second hydrolysis stage was performed after dilution to $2.6 \%$ acid concentration at $120{ }^{\circ} \mathrm{C}$ and $0.12 \mathrm{MPa}$ for 30 minutes. The content of glucose and xylose was determined by means of acid methanolysis followed by gas chromatography for MFC, and by Borat-AEC of sulfuric acid hydrolysates for all other variants, respectively [22]. The crystallinity of cellulose was estimated according to Ref. [23] from X-ray powder diffractograms obtained with a Rigaku SmartLab 5-Axis X-ray diffractometer using glass capillaries at ambient conditions. ATR-IR spectra were recorded from air-dried lignocellulose films with a Perkin Elmer FT-IR Spectrometer equipped with a Universal
ATR Sampling Accessory. Each sample was scanned in quadruplicate from 650 to $4000 \mathrm{~cm}^{-1}$ with a resolution of $4 \mathrm{~cm}^{-1}$ for 32 times. XPS was done with a K-Alpha spectrometer (Thermo Fischer Scientific). Survey scans were done with a pass energy of $200 \mathrm{eV}$ and a step size of $1.0 \mathrm{eV}$. High resolution scans were done with a pass energy of $50 \mathrm{eV}$ and a step size of $0.1 \mathrm{eV}$.

Air-dried films of all fibrillated materials were placed on aluminium specimen holders, sputter coated with a platinum layer of about $7.5 \mathrm{~nm}$ (BALTEC MED 020 Modular High Vacuum Coating Systems, BAL-TEC AG, Liechtenstein) using Ar as a carrier gas at 0.05 mbar and SEM was carried out using a FEI Nova NanoSEM 230 instrument (FEI, Hilsboro, Oregon, USA) at an accelerating voltage of $5 \mathrm{kV}$ and a working distance of $5 \mathrm{~mm}$. All AFM measurements were done in quantitative nanomechanical mapping (QNM) mode with a Dimension Icon AFM (Bruker AXS, France; formerly Veeco) recording topography and tip-to-surface adhesion amongst other properties. In QNM-mode force-distance-curves are evaluated for each pixel to give information on tip-to-surface mechanical interactions. Air dry films and individual fibrils prepared by depositing a drop of fibril suspension of $0.001 \%$ fibril content in water onto mica, and subsequent evaporation of water at ambient conditions were investigated. All scans were performed using Bruker's ScanAsyst-Air probes $\left(k=0.4 \mathrm{~N} / \mathrm{m}, f_{0}=70 \mathrm{~Hz}\right.$, $r=2 \mathrm{~nm}$; nominal values, probe-specific data calibrated before scans). To keep the tip speed constant scan rates of 0.1 and $0.5 \mathrm{~Hz}$ where chosen in accordance to the scan sizes of 5 and $1 \mu \mathrm{m}$. Tip frequency was set to $1 \mathrm{kHz}$ and peak-force set-point to $0.5 \mathrm{nN}$. All images were evaluated using Gwyddion Image Analysis (V2.39) freeware.

\section{Preparation of PLA composites}

Fibrillated material was dehydrated by means of solvent exchange from water to ethanol and 
Figure 1 ATR FT-IR spectra of different variants of fibrillated material.

Representative spectral regions indicating variability in lignin and hemicellulose (xylan) content are indicated.

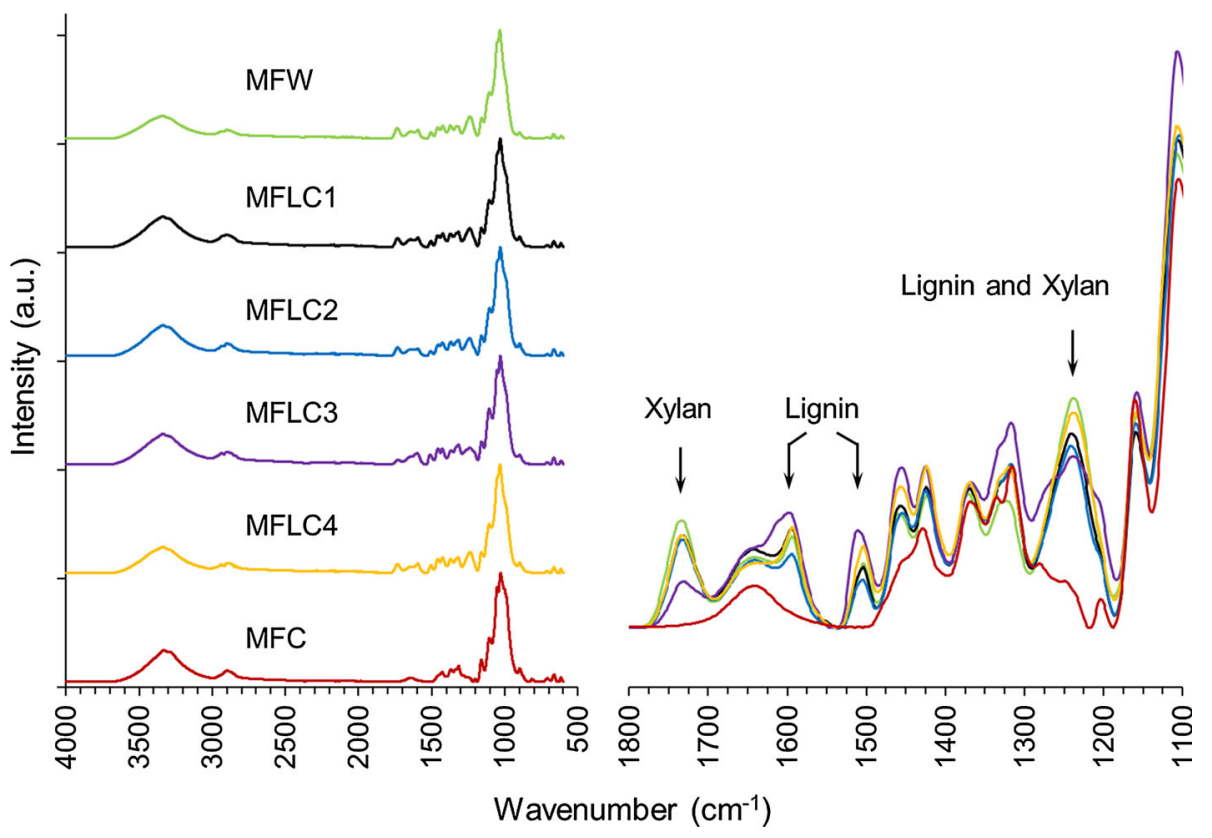

Results and discussion

isopropanol (3 repetitions each) and subsequently dried until mass constance at $103{ }^{\circ} \mathrm{C}$. For the preparation of composite films by means of casting from solution, $10 \mathrm{~g}$ PLA (Ingeo Biopolymer 3251D purchased from NatureWorks) was dissolved in $100 \mathrm{ml}$ chloroform. An aliquot of dry fibrillary material was added to the solution to achieve a target fibre loading in the final composite of $1 \%(\mathrm{w} / \mathrm{w})$ and dispersed with an Ultra Turrax device for 2 minutes. Thereafter, the dispersion was poured into a Petri dish, covered with tissue paper, and the solvent was left to evaporate overnight, resulting in films with $0.1 \mathrm{~mm}$ thickness. To remove any remaining solvent the films were subjected to additional vacuum drying at $40{ }^{\circ} \mathrm{C}$ and 80 mbar.

\section{Characterisation of PLA composites}

The macroscopic dispersion of filler in the PLA films was evaluated by transmitted light microscopy. Tensile mechanical properties of the films were evaluated with a Zwick-Roell $20 \mathrm{kN}$ universal testing machine equipped with a $500 \mathrm{~N}$ load cell. For this purpose, parallel strips with a length of $50 \mathrm{~mm}$ and a width of $8 \mathrm{~mm}$ were cut from the thin films and tested at a free span length of $30 \mathrm{~mm}$, and a rate of $1 \mathrm{~mm} \mathrm{~min}{ }^{-1}$, without using end tabs glued to the end of the strips. The modulus of elasticity of the specimens was calculated using the machine deformation as indicative of sample deformation. 20 specimens were evaluated for each variant.

\section{Chemical composition, morphology, and surface chemistry}

The samples investigated in the present study were chosen with the aim of representing a high degree in variability of the content of cell wall polymers. In this context, the advantage of the mild ethanol/water (organosolv) delignification procedure chosen in the present study is that no inorganic elements or charges are introduced into the substrate, which limits specimen variability to variability in the content and structure of natural cell wall constituents. ATR FT-IR spectra shown in Fig. 1 demonstrates that the aim of obtaining high chemical variability between the treatments MFLC1 and MFLC4 was fully achieved. On the one hand, aromatic skeletal vibrations at 1595 and $1505 \mathrm{~cm}^{-1}$ [24] as well as syringyl ring breathing at $1242 \mathrm{~cm}^{-1}$ [25] indicate highly variable lignin content. On the other hand, the unconjugated $\mathrm{C}=\mathrm{O}$ band at $1733 \mathrm{~cm}^{-1}$ [24] and $\mathrm{C}-\mathrm{O}$ stretching at $1242 \mathrm{~cm}^{-1}$ [25] reveal variable hemicellulose, specifically xylan, content. In good agreement with apparent chemical variability, structural variability in terms of fraction of crystalline material present in the fibrils is revealed by XRD (Fig. 2; Table 2). A detailed analysis of peak width of the different reflections attributed to the cellulose I crystal did not reveal any measurable change in terms of crystallite size due to the different treatments applied. Thus it may be 


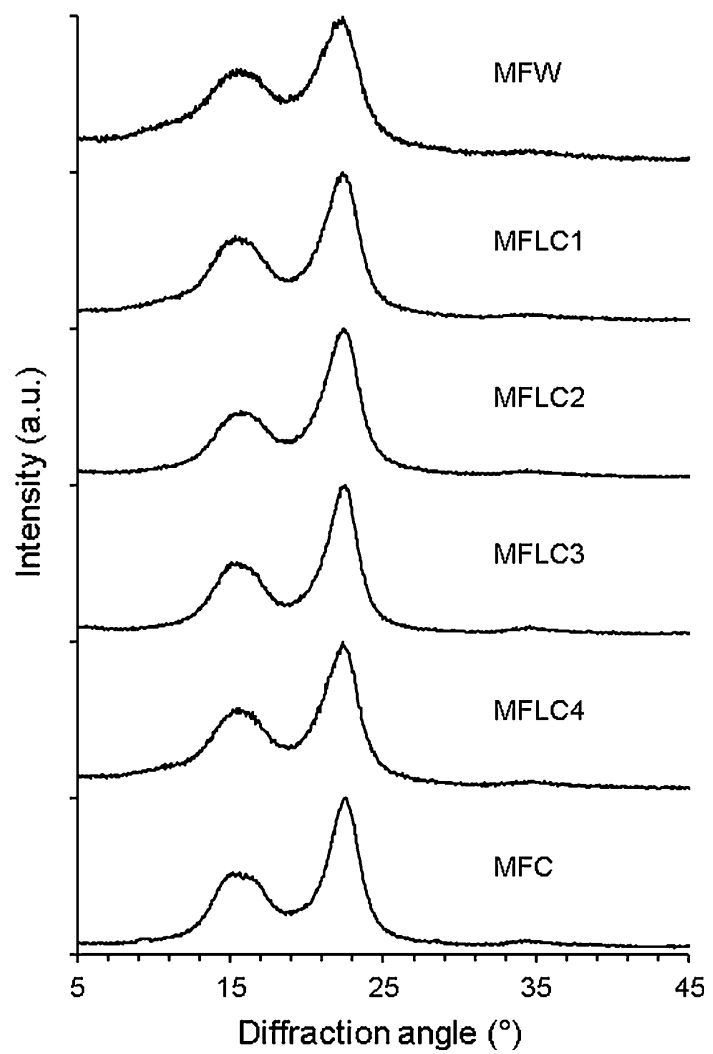

Figure 2 X-ray powder diffractograms of different microfibrillated cellulose samples.

concluded that the variability in crystallinity of the specimens is not due to variability in cellulose crystallinity per se, but simply due to the fact that cellulose content is highly variable in the different variants, which also entails variability in overall sample crystallinity.

Wet-chemical analysis of the most relevant chemical constituents in the variants of fibrillated material produced (Table 2) confirms the first assessment of chemical variability by means of ATR FT-IR. Microfibrillated wood (MFW) prepared from untreated beech wood and microfibrillated cellulose (MFC) may be considered as the two extreme variants, between which the other for variants of microfibrillated lignocellulose (MFLC) prepared from beech wood delignified to different extent are located in terms of chemical composition. As shown in Table 2, a broad range in variability was obtained regarding all major cell wall polymers. Lignin content is highest in MFW, while the MFLC variants show intermediate lignin content and MFC is virtually free of lignin. Lignin content decreases progressively from variant MFLC1 to MFLC3, corresponding to the increasing severity of treatment due to addition of sulfuric acid. The variant MFCL4, which was subjected to identical conditions as MFLC1 but shorter treatment time, shows only slightly higher lignin content than MFLC1. The content of xylose, which is indicative of the most important hardwood hemicellulose xylan, is closely correlated with lignin content, particularly in the partially delignified MFLC specimens. By contrast, the content of cellulose is inversely correlated with lignin content. MFC is very pure cellulose, and also MFLC 3 contains a high amount of cellulose.

\section{Morphology and surface chemical characteristics}

The morphology of the different fibrillated specimens was examined by means of scanning electron microscopy (SEM, Fig. 3). In all specimens a significant proportion of only partially fibrillated material, which is particularly obvious in the MFLC samples (Fig. 3b-e), and to a lesser extent in MFW (Fig. 3a) and MFC (Fig. 3f), was found. Images taken at higher magnification show fibril aggregates with diameters well below $1 \mu \mathrm{m}$ in all types of specimens examined. Thus the material examined is termed "microfibrillated" in view of the prevalence of micron and submicron fibril dimensions.

AFM images taken from the same dry film specimens observed with SEM reveal fibrillary structures in all types of fibrillated material examined (Fig. 4). Typical fibril diameters are in the order of $50 \mathrm{~nm}$, and
Table 2 Chemical composition $(\% \mathrm{w} / \mathrm{w})$ and crystallinity index of the different fibre materials used

\begin{tabular}{llllll}
\hline Specimen type & Glucose & Xylose & Mannose & Lignin & Crystallinity \\
\hline MFW & 41.0 & 17.8 & 1.0 & 25.1 & 0.53 \\
MFLC1 & 56.2 & 16.0 & 1.3 & 17.0 & 0.66 \\
MFLC2 & 62.0 & 14.1 & 1.4 & 14.0 & 0.71 \\
MFLC3 & 80.6 & 8.7 & 1.1 & 8.5 & 0.74 \\
MFLC4 & 53.4 & 17.4 & 1.3 & 17.3 & 0.65 \\
MFC & 99.7 & 0.0 & 0.0 & 0.3 & 0.70 \\
\hline
\end{tabular}



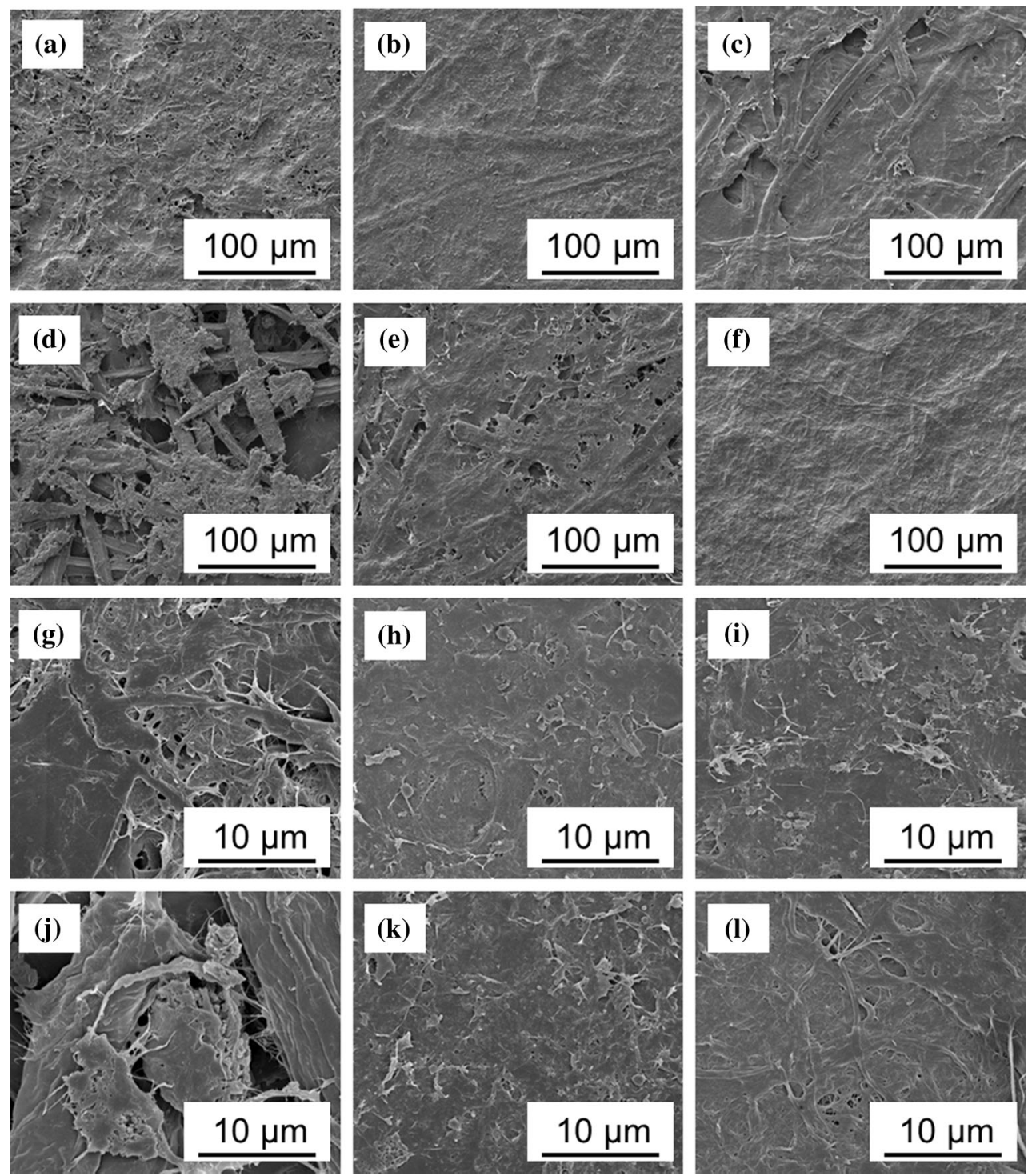

Figure 3 Representative SEM images of dried films of fibrillated material taken at two different magnifications. Images a-f show specimens MFW, MFLC1-4, and MFC, respectively, at low

finer structures are seldom observed, with the exception of MFC. It may be assumed that the noncellulosic cell-wall components, which are present in comparative abundance, are responsible for the rather closed surface character observed, which was also proposed for similar material examined in other studies [13, 14]. While the differences in surface topography discernible in AFM height images are limited in the case of dry films, average AFM adhesion forces recorded for these samples reveal high variability in adhesion forces between the individual films (Figs. 4, 5). The interpretation of adhesion forces (Fig. 5a) measured on a rough and chemically magnification, whereas images $\mathbf{g}-\mathbf{1}$ show the same specimens at high magnification.

heterogeneous surface such as specimens used in the present study is complex. Nonetheless, even in humid conditions the measured pull-off force, which is dominated by capillary forces, contains surfacespecific information [26]. Surface roughness is an additional parameter which may affect adhesion forces. However, no such effect was obvious in our data (Fig. 5b). As shown in Fig. 5a, the mean value of adhesion forces is different for the samples characterised, and also the amount of variability is different. Clearly, variability is lowest in MFC, which agrees with the chemical homogeneity of this specimen consisting essentially only of cellulose. In contrast, 

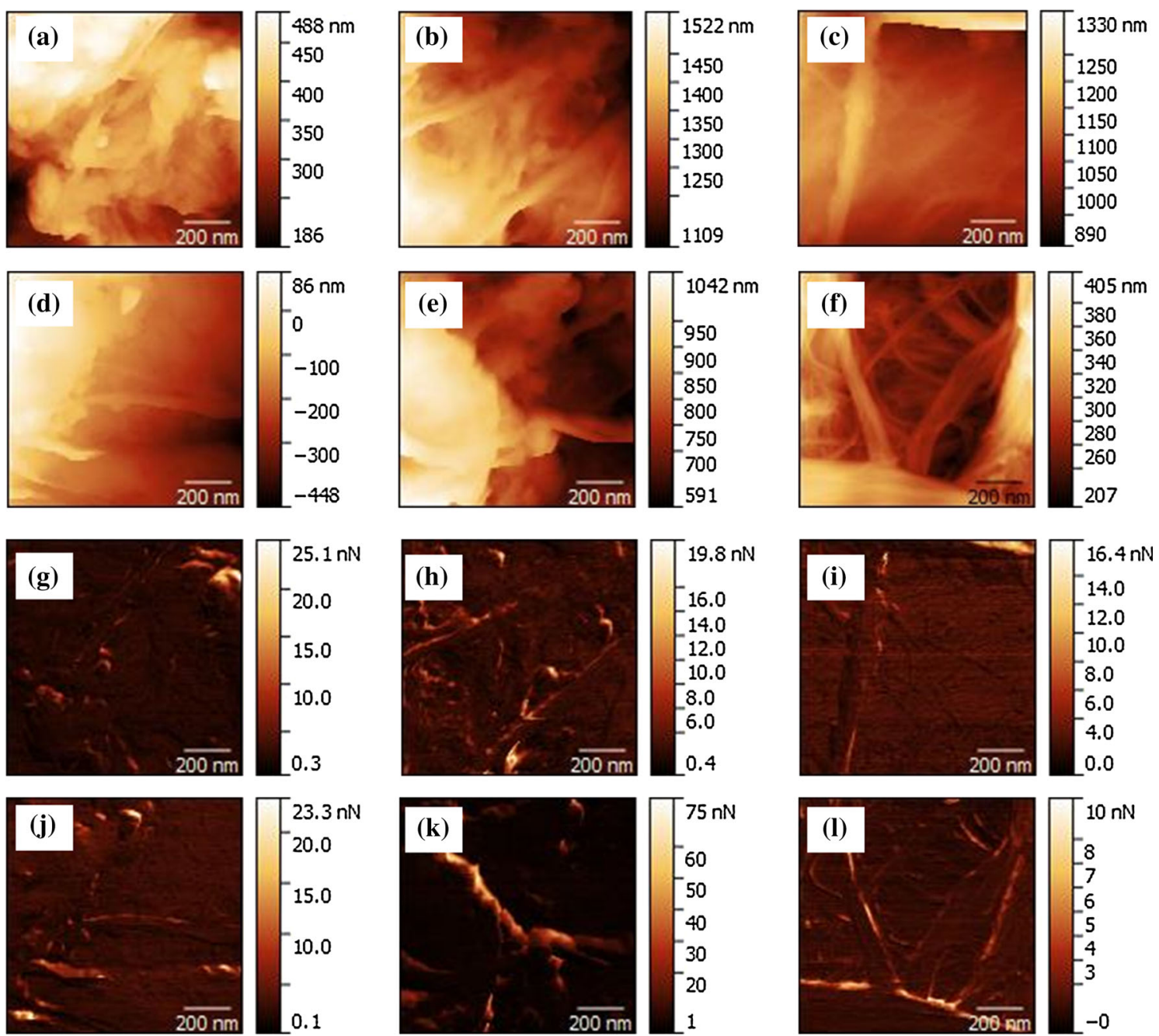

Figure 4 Representative AFM images of specimen regions used for the analysis of AFM tip adhesion force distribution. Images a-f show topography and images $\mathbf{g}-\mathbf{1}$ show the corresponding

the variability of adhesion forces is high in the MFW and MFLC samples, with maximum values for MFLC3, reflecting the chemical heterogeneity of these specimens. Thus, variability of surface properties in terms of adhesion forces measured is high within one sample. However, this does not mean that averaged adhesion force suffers from little significance due to high variability. In fact, such measurements are highly reproducible, which is confirmed by coefficients of variation $<4 \%$ observed for the averaged adhesion force determined with consecutive measurements in different regions of one sample.

Surface chemical variability observed by adhesion force measurements is confirmed by XPS analysis (Table 3). The samples measured clearly differ in their content of carbon and oxygen, and in the ratio of adhesion force maps. The series $\mathbf{a}-\mathbf{f}$ and $\mathbf{g}-\mathbf{l}$ each correspond to specimens MFW, MFLC1-4, and MFC, respectively.

non-polar and polar moieties. Values for the $\mathrm{C} / \mathrm{O}$ ratio of beech wood found in literature vary between 1.81 [27] and 2.22 [28]. Heat treatment of beech wood, which leads to surface inactivation due to loss in polar character, entails an increase in $\mathrm{C} / \mathrm{O}$ to 2.27 [27]. Thus it is presumed that increasing $\mathrm{C} / \mathrm{O}$ ratios in the samples characterised in the present study indicate a progressive change of surface polarity. Surprisingly, no obvious relationship or clear correlations between bulk chemistry (Table 2) and surfacerelated XPS data (Table 3) were found. This may be explained by the obvious observation that XPS is surface specific and not representative of the bulk of the sample. It was shown, for example, that surface lignin content of various pulp fibres can be determined from changes in the $\mathrm{C} / \mathrm{O}$ ratio measured with 
Table 3 XPS results for nanopapers produced from different fibrillated materials

\begin{tabular}{lccllcc}
\hline Specimen type & $\mathrm{O} 1 \mathrm{~s}$ & $\mathrm{C} 1 \mathrm{~s}$ & $\mathrm{C}-\mathrm{C}, \mathrm{C}-\mathrm{H}$ & $\mathrm{C}-\mathrm{O}-\mathrm{C}, \mathrm{OH}$ & $\mathrm{C}=\mathrm{O}, \mathrm{O}-\mathrm{C}-\mathrm{O}$ & $\mathrm{COOH}$ \\
\hline MFW & 34.1 & 64.7 & 29.7 & 58.5 & 9.8 & 2.0 \\
MFLC1 & 35.0 & 64.3 & 26.2 & 61.5 & 10.6 & 1.6 \\
MFLC2 & 35.3 & 64.0 & 24.8 & 62.4 & 10.7 & 1.9 \\
MFLC3 & 32.6 & 67.4 & 33.8 & 56.5 & 8.6 & 0.9 \\
MFLC4 & 33.6 & 64.4 & 28.9 & 59.9 & 9.2 & 1.9 \\
MFC & 38.6 & 60.7 & 16.8 & 68.4 & 14.0 & 0.9 \\
\hline
\end{tabular}

XPS [29]. Dissolved lignin may precipitate on pulp fibres during the washing phase of the organosolv approach to delignification used in the present study [30]. AFM images of MFC and MFLC (Fig. 6) show evidence for precipitation of lignin in terms of the surface morphology of MFLC. While MFC fibrils appear smooth, MFLC is characterised by the
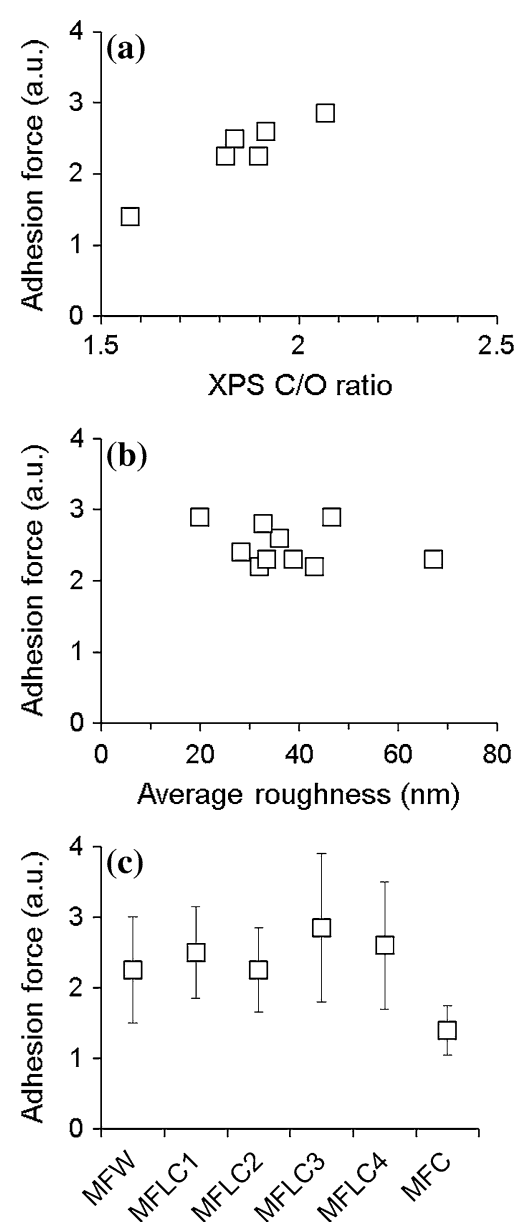

Figure 5 Average valued and standard deviation of adhesion forces recorded for different types of fibrillated material (a) and relationship between adhesion forces measured and the surface roughness of specimens (b) as well as correlation of adhesion forces with XPS results (c). occurrence of numerous fine granular structures along the fibril surface, which are presumably lignin residues [15].

By contrast to the reported lack of correlation between bulk- and surface chemistry, respectively, a clear relationship between the increasing carbon/ oxygen ratio determined with XPS, and increasing AFM adhesion force is found (Fig. 5c). One the one hand, this is not unexpected since both analytical methods are highly surface specific. On the other hand, the significance of AFM adhesion force measurements in terms of surface chemistry is not always obvious. However, within the boundaries set by the experimental conditions of the present study, a clear correlation between increasing $\mathrm{C} / \mathrm{O}$ ratio and increasing adhesion force shown in Fig. $5 \mathrm{c}$ is evident.

\section{Dispersion and reinforcement effect of fibrils in PLA}

Already during film casting it was obvious that the different variants of fibrils show different dispersion in the chloroform/PLA solution. MFC quickly tended to agglomerate, forming white cellulose grains in the liquid (Fig. 7). By contrast, MFLC and MFW showed more stable and homogeneous dispersion. The resulting dispersion of fibrils in PLA films is shown in Fig. 8. MFW (Fig. 8a) is evenly dispersed, but large unfibrillated particles are conspicuous. MFLC1-4 (Fig. 8b-e) show variable homogeneity of dispersion, with the best result achieved for variant MFLC3, and distinct agglomerates for MFC (Fig. 8f). Agglomeration of unmodified MFC upon addition to PLA/ chloroform and its deteriorating effect on composite properties is widely observed but efficiently tackled by e.g., surface acetylation [31, 32]. Similar to bulkand surface chemistry as well as dispersion, also the mechanical performance in tensile tests reveals a broad variation of results for the filler variants investigated (Table 4). It should be noted that the mechanical characterisation performed here is not 
Figure 6 AFM tapping mode height (a, c) and phase (b, d) images of $\operatorname{MFC}(\mathbf{a}, \mathbf{b})$ and $\operatorname{MFLC}(\mathbf{c}, \mathbf{d})$.
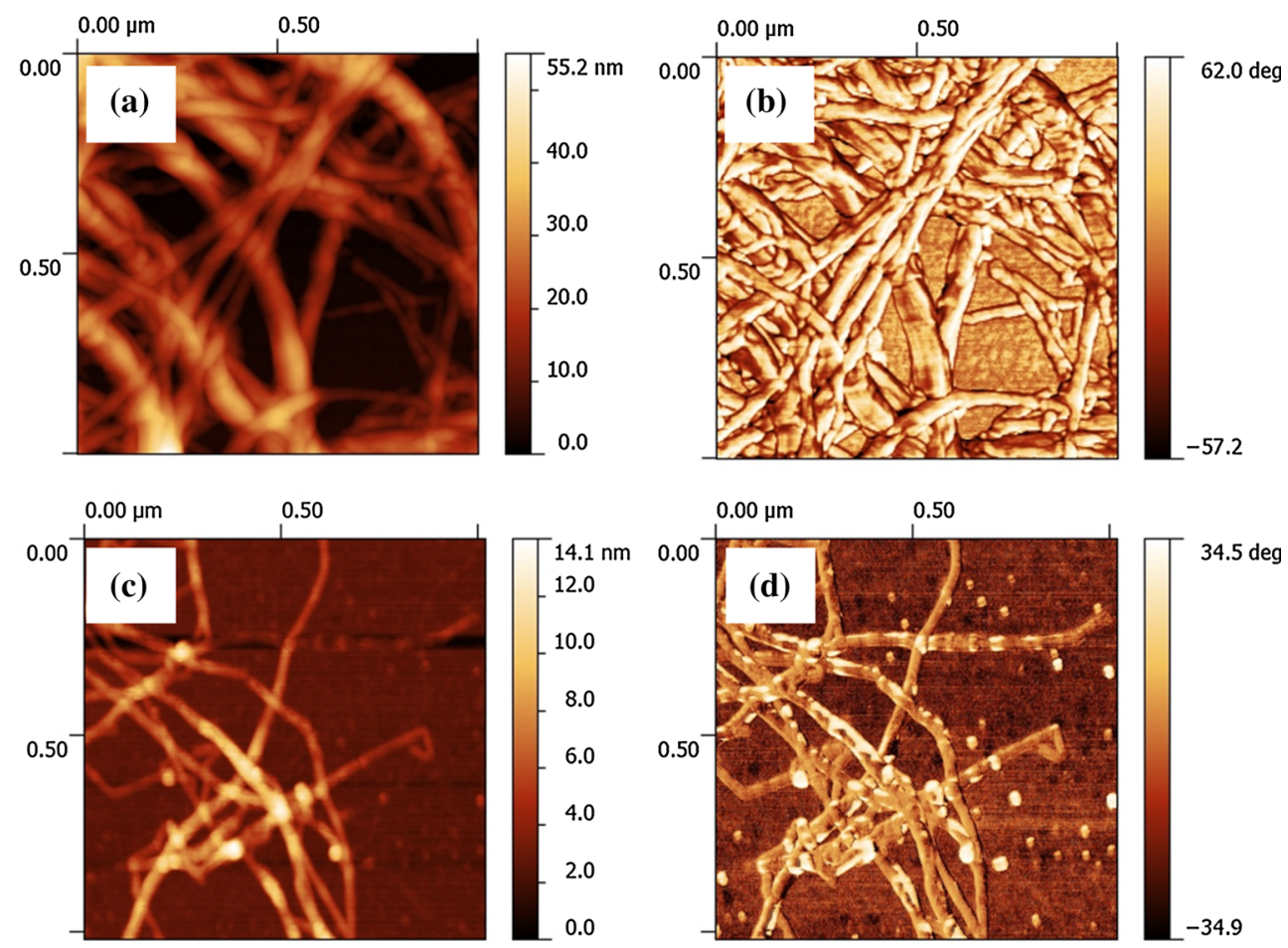
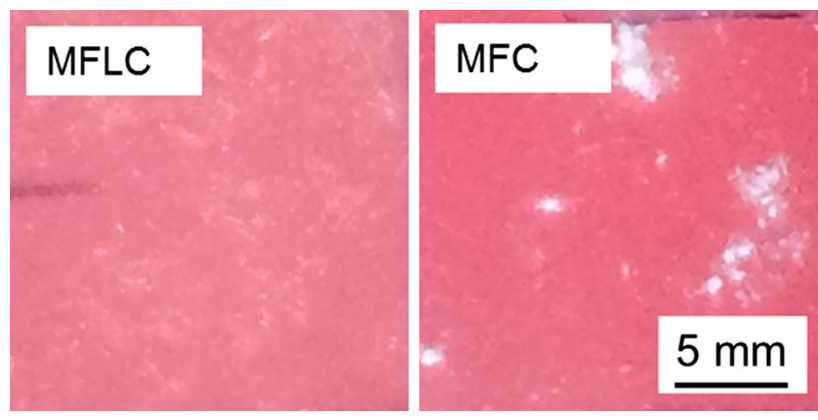

Figure 7 Exemplary incident light photograph of filler dispersion for MFLC in PLA compared to MFC taken against red background highlighting agglomeration of white MFC.

intended to serve as a valid evaluation of polymer reinforcement. To fulfil this goal, standard processing conditions such as melt compounding at variable filler content would be required. Also, PLA disposes of strong mechanical performance also in unreinforced state [1], which is why higher filler content would probably be required to produce significant effects. The PLA films produced by casting from solution in the present study purely serve to assess dispersion of different filler variants in a non-polar medium. Notably, tensile strength is sensitive to inhomogeneities in filler dispersion and thus a suitable characterisation parameter. Furthermore it is obvious that the absolute values of mechanical properties shown here differ from melt-compounded PLA in that strength and modulus are inferior, which is due to differences in crystallisation behaviour [33] and potentially also due to small amounts of residual solvent. Nonetheless, the results shown in Table 4 are presumed valid for the purpose of comparison within the present study. Overall, the addition of $1 \%$ microfibrillated material to PLA resulted in an improvement of tensile properties in terms of strength and modulus of elasticity, in parallel to a reduction in extensibility for most variants (Table 4). This effect is widely observed [1]. Only addition of MFW leads to reduced mechanical performance of PLA, presumably due to the presence of substantial unfibrillated fractions of material in this variant, as shown in Fig. 8a. To realise the full mechanical reinforcement efficiency of lignocellulosic material, a high degree of fibrillation is required in the first place [35]. Given that a material is fibrillated it is furthermore necessary that fibrillar structure is preserved during compounding, which is a major challenge in melt-compounding of MFC-reinforced PLA [35-38]. As shown in Fig. 8f, MFC, which is the most-fibrillated according to SEM images (Fig. 3f, 1), suffered from collapse during compounding with the PLA/ chloroform solution. Therefore, the full reinforcement potential of MFC was not realised in the cast PLA 

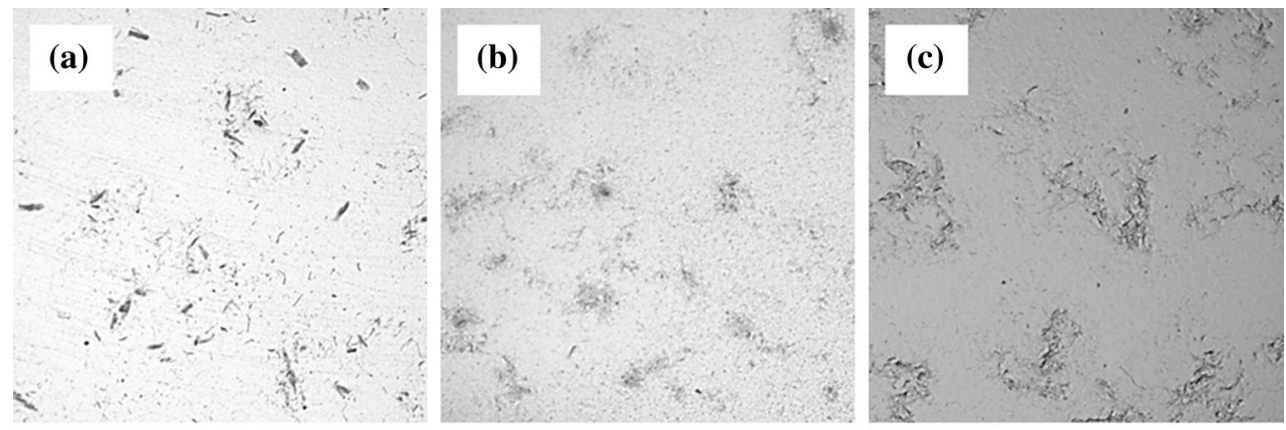

\section{(d)}

(e)

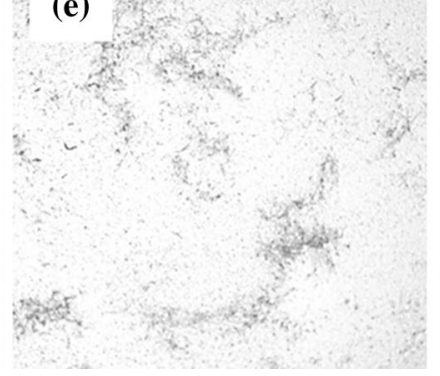

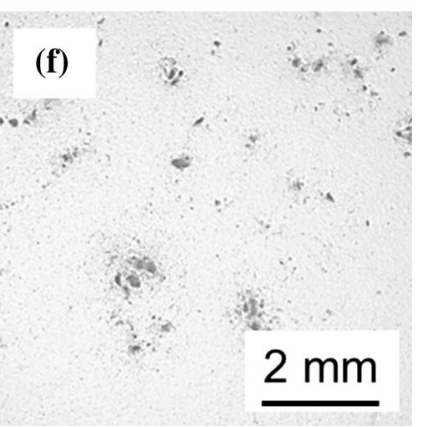

Figure 8 Filler dispersion in PLA by transmitted light microscopy: a MFW, b-e MFLC1-4, f MFC.

Table 4 Tensile properties of different variants of composite films (average values and standard deviation)

\begin{tabular}{lclc}
\hline Specimen type & Modulus of elasticity (MPa) & Tensile strength (MPa) & Elongation at break (\%) \\
\hline PLA & $902 \pm 58$ & $27.0 \pm 08$ & $13.8 \pm 3.5$ \\
MFW & $872 \pm 44$ & $25.3 \pm 1.0$ & $11.2 \pm 1.8$ \\
MFLC1 & $1153 \pm 96$ & $32.2 \pm 1.8$ & $5.5 \pm 0.7$ \\
MFLC2 & $995 \pm 108$ & $27.4 \pm 2.2$ & $8.2 \pm 3.0$ \\
MFLC3 & $1233 \pm 55$ & $35.2 \pm 2.5$ & $4.9 \pm 0.6$ \\
MFLC4 & $1077 \pm 73$ & $30.1 \pm 1.8$ & $6.6 \pm 1.1$ \\
MFC & $1075 \pm 134$ & $29.4 \pm 2.5$ & $5.8 \pm 0.8$ \\
\hline
\end{tabular}

films (Table 4), even though solely based on its high degree of fibrillation and high cellulose content compared to all other variants, high reinforcement should be expected. Same as with macroscopic dispersion of filler, the variant MFLC3 also performs best in terms of reinforcement of PLA. To find an explanation for this observation the results of mechanical characterisation were analysed in terms of eventual correlations with bulk- and surface chemical properties of fibrillated material. While no clear relationships were evident for bulk chemistry, surface chemistry in terms of XPS C/O ratio and averaged AFM adhesion force showed a clear correlation with tensile strength (Fig. 9). Assuming that surface chemistry governs filler dispersion in a polymer solution, XPS and averaged AFM adhesion forces provide a tool for understanding surfacechemical filler-matrix interaction. MFC is an exception regarding the correlation shown in Fig. 9, as it shows better reinforcement than some other variants despite exhibiting highest polarity based on $\mathrm{C} / \mathrm{O}$ ratio. Apparently the high reinforcement potential of MFC, which is pure, highly fibrillated cellulose, still provides some strength improvement despite obviously inferior dispersion in PLA.

In summary, surface-chemical compatibility of fibrillated lignocellulosic filler with PLA was improved by the presence of residual cell-wall polymers in certain variants. This lead to improved dispersion in cast PLA films and improved tensile performance. MFLC may thus be a viable option for producing certain natural fibre reinforced polymers with reasonable mechanical performance without performing chemical surface modification. While residual cell wall polymers do not provide MFC with hydrophobisation similar to much more efficient 


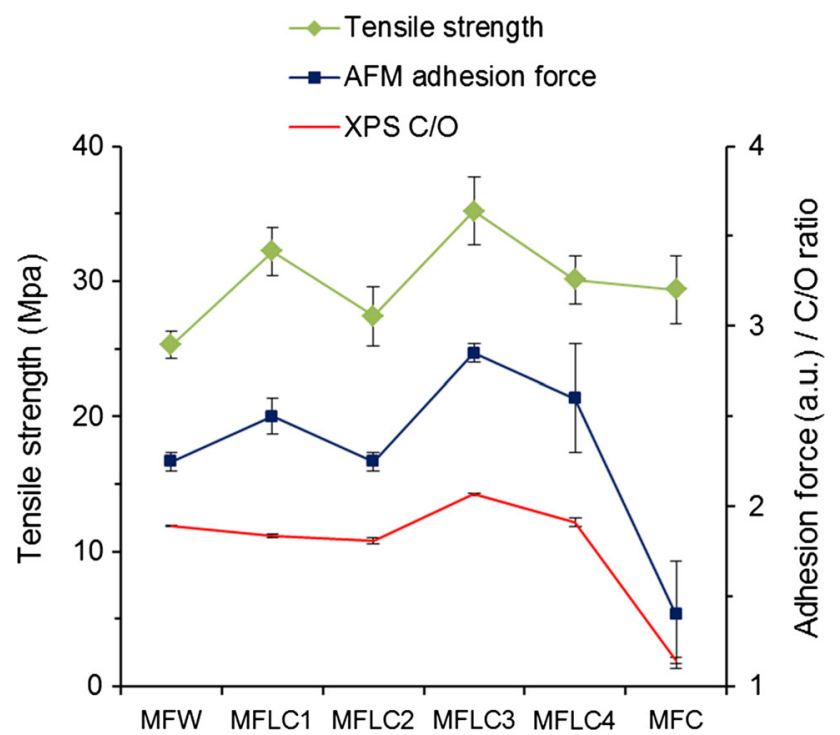

Figure 9 Variability of average surface-chemical characteristics related to tensile strength of PLA composite films. Average values from duplicate determinations are shown for XPS and AFM adhesion force, whereas tensile strength was calculated from 20 specimens tested each. Error bars represent standard deviation.

chemical modification, a limited degree of compatibility with non-polar polymer was demonstrated in the present study.

\section{Conclusion}

The results presented above contribute to a better understanding of the role of cell-wall polymers in providing surface-chemical compatibility with nonpolar polymers. In particular, it was shown that partially de-lignified material may show distinctly reduced surface polarity. Notably, the reduction in surface polarity does not necessarily correlate with bulk chemical composition. Furthermore it was shown that reduced surface polarity leads to improved dispersion of fibrillated material in a nonpolar, which in turn benefits the mechanical performance of a composite material. With the exception of MFC, which stands out by high degree of fibrillation compared to all other variants studied, AFM adhesion forces measured on filler surfaces showed good correlation with strength values of composites reinforced with the respective fillers. AFM adhesion forces may thus be a useful tool for characterising fillermatrix interactions in lignocellulose-reinforced polymers.

\section{Acknowledgements}

Open access funding provided by University of Natural Resources and Life Sciences Vienna (BOKU). The authors declare that no funding was received for this study.

\section{Compliance with ethical standards}

Conflict of interest The authors declare that they have no conflict of interest.

Open Access This article is distributed under the terms of the Creative Commons Attribution 4.0 International License (http://creativecommons.org/licen ses/by/4.0/), which permits unrestricted use, distribution, and reproduction in any medium, provided you give appropriate credit to the original author(s) and the source, provide a link to the Creative Commons license, and indicate if changes were made.

\section{References}

[1] Lee KY, Aitomaki Y, Berglund LA, Oksman K, Bismarck A (2014) On the use of nanocellulose as reinforcement in polymer matrix composites. Compos Sci Technol 105:15-27

[2] Eichhorn SJ, Dufresne A, Aranguren M, Marcovich NE, Capadona JR, Rowan SJ, Weder C, Thielemans W, Roman M, Renneckar S, Gindl W, Veigel S, Keckes J, Yano H, Abe K, Nogi M, Nakagaito AN, Mangalam A, Simonsen J, Benight AS, Bismarck A, Berglund LA, Peijs T (2010) Review: current international research into cellulose nanofibres and nanocomposites. J Mater Sci 45:1-33. doi:10. 1007/s10853-009-3874-0

[3] Klemm D, Kramer F, Moritz S, Lindstrom T, Ankerfors M, Gray D, Dorris A (2011) Nanocelluloses: a new family of nature-based materials. Angew Chem Int Ed 50:5438-5466

[4] Dufresne A (2013) Nanocellulose: a new ageless bionanomaterial. Mater Today 16:220-227

[5] Mazeau K (2011) On the external morphology of native cellulose microfibrils. Carbohydr Polym 84:524-532

[6] Habibi Y (2014) Key advances in the chemical modification of nanocelluloses. Chem Soc Rev 43:1519-1542

[7] Terashima N, Kitano K, Kojima M, Yoshida M, Yamamoto H, Westermark U (2009) Nanostructural assembly of cellulose, hemicellulose, and lignin in the middle layer of secondary wall of ginkgo tracheid. J Wood Sci 55:409-416

[8] Iwamoto S, Abe K, Yano H (2008) The effect of hemicelluloses on wood pulp nanofibrillation and nanofiber network characteristics. Biomacromolecules 9:1022-1026 
[9] Galland S, Berthold F, Prakobna K, Berglund LA (2015) Holocellulose nanofibers of high molar mass and small diameter for high-strength nanopaper. Biomacromolecules 16:2427-2435

[10] Gu J, Hsieh YL (2015) Surface and structure characteristics, self-assembling, and solvent compatibility of holocellulose nanofibrils. ACS Appl Mater Interfaces 7:4192-4201

[11] Jiang F, Hsieh YL (2015) Holocellulose nanocrystals: amphiphilicity, oil/water emulsion, and self-assembly. Biomacromolecules 16:1433-1441

[12] Abe K, Nakatsubo F, Yano H (2009) High-strength nanocomposite based on fibrillated chemi-thermomechanical pulp. Compos Sci Technol 69:2434-2437

[13] Rojo E, Peresin MS, Sampson WW, Hoeger IC, Vartiainen J, Laine J, Rojas OJ (2015) Comprehensive elucidation of the effect of residual lignin on the physical, barrier, mechanical and surface properties of nanocellulose films. Green Chem 17:1853-1866

[14] Herzele S, Veigel S, Liebner F, Zimmermann T, Gindl-Altmutter W (2015) Reinforcement of polycaprolactone with microfibrillated lignocellulose. Ind Crops Prod. doi:10.1016/ j.indcrop.2015.12.051

[15] Gindl-Altmutter W, Obersriebnig M, Veigel S, Liebner F (2015) Compatibility between cellulose and hydrophobic polymer provided by microfibrillated lignocellulose. ChemSusChem 8:87-91

[16] Wang X, Cui X, Zhang L (2012) Preparation and characterization of lignin-containing nanofibrillar cellulose. Procedia Environ Sci 16:125-130

[17] Wang X, Sun HB, Bai HL, Zhang LP (2014) Thermal, mechanical, and degradation properties of nanocomposites prepared using lignin-cellulose nanofibers and poly(lacticacid). Bioresources 9:3211-3224

[18] Ballner D, Herzele S, Keckes J, Edler M, Griesser T, Saake B, Liebner F, Potthast A, Paulik C, Gindl-Altmutter W (2016) Lignocellulose nanofiber-reinforced polystyrene produced from composite microspheres obtained in suspension polymerization shows superior mechanical performance. ACS Appl Mater Interfaces 8:13520-13525

[19] Nampoothiri KM, Nair NR, John RP (2010) An overview of the recent developments in polylactide (PLA) research. Bioresour Technol 101:8493-8501

[20] Puls J, Schreiber A, Saake B (2009) Conversion of beechwood into platform chemicals after organosolv treatment. In: 15th International Symposium on Wood, Fibre and Pulping Chemistry, Oslo, Norway

[21] Muurinen E (2000) Organosolv pulping. A review and distillation study related to peroxyacid pulping. Academic Dissertation, Faculty of Technology, University of Oulu, Finland, 314 pp
[22] Willfor S, Pranovich A, Tamminen T, Puls J, Laine C, Suurnakki A, Saake B, Uotila K, Simolin H, Hemming J, Holmbom B (2009) Carbohydrate analysis of plant materials with uronic acid-containing polysaccharides-A comparison between different hydrolysis and subsequent chromatographic analytical techniques. Ind Crops Prod 29:571-580

[23] Segal L, Creely J, Martin A, Conrad C (1959) An empirical method for estimating the degree of crystallinity of native cellulose using the X-ray diffractometer. Text Res J 29:789-794

[24] Rodrigues J, Faix O, Pereira H (1998) Determination of lignin content of Eucalyptus globulus wood using FTIR spectroscopy. Holzforschung 52:46-50

[25] Popescu CM, Popescu MC, Singurel G, Vasile C, Argyropoulos DS, Willfor S (2007) Spectral characterization of eucalyptus wood. Appl Spectrosc 61:1168-1177

[26] Sedin DL, Rowlen KL (2000) Adhesion forces measured by atomic force microscopy in humid air. Anal Chem 72:2183-2189

[27] Inari GN, Petrissans M, Lambert J, Ehrhardt JJ, Gerardin P (2006) XPS characterization of wood chemical composition after heat-treatment. Surf Interface Anal 38:1336-1342

[28] Sinn G, Reiterer A, Stanzl-Tschegg SE (2001) Surface analysis of different wood species using X-ray photoelectron spectroscopy (XPS). J Mater Sci 36:4673-4680. doi:10. 1023/A:1017954300015

[29] Johansson LS, Campbell JM, Koljonen K, Stenius P (1999) Evaluation of surface lignin on cellulose fibers with XPS. Appl Surf Sci 144:92-95

[30] Xu Y, Li K, Zhang M (2007) Lignin precipitation on the pulp fibers in the ethanol-based organosolv pulping. Colloids Surf A 301:255-263

[31] Tingaut P, Zimmermann T, Lopez-Suevos F (2010) Synthesis and characterization of bionanocomposites with tunable properties from poly(lactic acid) and acetylated microfibrillated cellulose. Biomacromolecules 11:454-464

[32] Bulota M, Kreitsmann K, Hughes M, Paltakari J (2012) Acetylated microfibrillated cellulose as a toughening agent in poly(lactic acid). J Appl Polym Sci 126:E448-E457

[33] Suryanegara L, Nakagaito AN, Yano H (2009) The effect of crystallization of PLA on the thermal and mechanical properties of microfibrillated cellulose-reinforced PLA composites. Compos Sci Technol 69:1187-1192

[34] Nakagaito AN, Yano H (2004) The effect of morphological changes from pulp fiber towards nano-scale fibrillated cellulose on the mechanical properties of high-strength plant fiber based composites. Appl Phys A 78:547-552

[35] Iwatake A, Nogi M, Yano H (2008) Cellulose nanofiberreinforced polylactic acid. Compos Sci Technol 68:2103-2106 
[36] Jonoobi M, Mathew AP, Abdi MM, Makinejad MD, Oksman K (2012) A comparison of modified and unmodified cellulose nanofiber reinforced polylactic acid (PLA) prepared by twin screw extrusion. J Polym Environ 20:991-997

[37] Jonoobi M, Harun J, Mathew AP, Oksman K (2010) Mechanical properties of cellulose nanofiber (CNF) reinforced polylactic acid (PLA) prepared by twin screw extrusion. Compos Sci Technol 70:1742-1747

[38] Eyholzer C, Tingaut P, Zimmermann T, Oksman K (2012) Dispersion and reinforcing potential of carboxymethylated nanofibrillated cellulose powders modified with 1-hexanol in extruded poly(lactic acid) (PLA) composites. J Polym Environ 20:1052-1062 